\title{
Maternal Health Services as Determinant Factors for Low Birth Weight in public hospitals of Addis Ababa, Ethiopia: a Case-control Study.
}

Yonas Abebe ( $\sim$ yonasabebe3367@gmail.com )

Addis Ababa University

Maedot Kebede

Debre Berhan University

Tomas Getahun

Armauer Hansen Research Institute

Marekegn Habtamu

Kotebe Metropolitan University

\section{Behailu Tariku}

Debre Berhan University

Esubalew Tesfahun

Debre Berhan University

\section{Research Article}

Keywords: Low Birth Weight, Maternal Health Services, Case Control, Addis Ababa, Ethiopia

Posted Date: January 10th, 2022

DOI: https://doi.org/10.21203/rs.3.rs-1221463/v1

License: (1) This work is licensed under a Creative Commons Attribution 4.0 International License.

Read Full License 


\section{Abstract}

Background: The birth weight of a newborn has a substantial impact on infant mortality, morbidity, development, and long-term health. It is determined by the mother's overall health status. More than 20 million babies are born with low birth weight across the world. In developing countries, almost $17 \%$ of all newborns are born with low birth weights, with no exception in Ethiopia. Alleviating this problem, needs a clear understanding of the determinants. Thus, this study was done in Addis Ababa, the most populous city in the country, where the lifestyles of mothers might be affected by the dynamic city life.

Method: An unmatched case-control study was applied to assess the risk factors of low birth weight in three randomly selected public hospitals in Addis Ababa, Ethiopia. Data was collected through interviewer-administered structured questionnaires. Data analysis was done using SPSS version 24. Descriptive statistics using frequencies and percentages were used to describe the socio-demographic characteristics of the study participants. Bi-variable and multi-variable logistic regression analyses were used to assess the possible effect of determinant factors on low birth weight, with their respective odds ratios and $95 \%$ confidence intervals. P-values of less than 0.05 were considered statistically significant.

Result: We enrolled 168 cases and 336 controls; all completed the study with no refusal. Of all pregnancies, 90 (17.8\%) were unplanned and unwanted. And, $153(30.3 \%)$ of mothers have a history of prior abortion. Among the factors we studied, maternal age below 18 years (AOR: 2.69, 95\% Cl: 1.24, 5.84), unwanted and unplanned pregnancy (AOR: $1.25,95 \% \mathrm{Cl}: 1.09,2.66$ ), ANC visit in the last pregnancy below three (AOR: 3.23, 95\% Cl: 1.61, 6.49), female neonate (AOR: 1.09, 95\% Cl: 2.04, 3.20), not supplemented with iron and folic acid (AOR: $3.11,95 \% \mathrm{Cl}: 1.36,7.11$ ) and hematocrit level of < 30 (AOR: $2.29,95 \% \mathrm{Cl}: 1.00,5.22)$ showed a statistically significant association with low birth weight.

Conclusion: This study demonstrates that the age of mothers below 18 years, unwanted and unplanned pregnancy, low ANC visits, lack of iron and folic acid supplements, and low maternal hemoglobin level were the significant determinants of low birth weight among term babies. Thus, women of childbearing age should be screened and educated about the risks of pregnancy and should get the necessary care and support when they get pregnant.

\section{Background}

According to WHO definition, Low Birth Weight (LBW) is a birth weight of a live born infant equal to 2,499 gm or less, regardless of gestational age. Subcategories include very Low Birth Weight (VLBW), which is less than $1500 \mathrm{gm}$ and Extremely Low Birth Weight (ELBW), which is less than $1000 \mathrm{gm}$, while normal birth weight at term delivery is 2500 to $4200 \mathrm{gm}$ [1]. Globally, it is estimated that more than 20 million livebirths were suffered from low birth weight, which is equivalent to $15-20 \%$ of all live births worldwide.

Birth weight is an essential indicator of public health status of population. More than $95.0 \%$ low births occur in developing countries, of which nearly 9.3 million are in South Asia and above 3.1 million in sub- 
Saharan Africa [2, 3]. It is the most important predictor of infant mortality, particularly for deaths within the first month of life $[2,4]$. LBW remains a major public health issue around the world, notably in several sub-Saharan African nations, where the global and sub-Saharan African countries prevalence rates of $15.5 \%$ and $15 \%$ respectively $[3,5]$. These statistics are greater than the World summit for children's aims of not more than $10 \%$ of LBW incidence [3]. This suggests that LBW is a major public health concern [6].It also evidences scant progress and babies born at LBW face the most severe risks [7]; that contribute to 60 to $80 \%$ of all neonatal deaths [8].

Ethiopia has an infant mortality rate of 59/1000 live births [9], and as a sub Saharan African country with poor socio economic status, it is a country with a higher rate of low birth weight. Though some studies have revealed prevalence of low birth weight ranging from $6 \%$ to $10 \%$, the problem would have been much worth if areas not investigated had accessed to readymade data now $[10,11]$. it is reasonable to assume that because the country hosts a large proportions of the low birth weight magnitudes observed in the region. However, to give concrete evidence, the country has limited data on BW estimates as most deliveries take place at home, leading to highly biased maternal subjective inclusion of a "very small baby" in the reports, as well as the scarcity of available studies done thus far [12-13]. Low birth weight has been on the rise in Ethiopia for the past two decades [10]. In addition, approximately three out of every ten children delivered to mothers in Afar (30\%), Amhara (28\%), Somali (26\%), and Gambella (27\%) were very small at birth [14]. Furthermore, cross-sectional studies undertaken at poket level across the country demonstrate that the prevalence of LBW varies widely from $11.2 \%$ in the north (Gondar) [12], to $22.5 \%$ in the south west (Jimma) [13]. The research implies that LBW is a substantial public health issue in the country, but one that has received little attention.

Along with the above facts, the researcher assumed that LBW was an indicator of a multifaceted publichealth problem that includes long-term maternal malnutrition, ill health, and poor health care in pregnancy and might be associated with the demographic, fertility, and reproductive history, as well as socio economic status of mothers, which could further be affected by the increasing burden of life in cities. Therefore, this study was conducted with the aim of providing an avenue for further new studies to focus on specific settings in order to identify contextualized determinants of this poor person's major public health problem. It will be a first step to provide evidence based context specific determinants of low birth weight to health programmers. In particular, evaluating the link between the determinant factors and low birth weight may be important for improving care for women who are at risk of low birth weight. It also provided development practitioners and policy makers with better information about where to go, and it may raise awareness about low birth weight among pregnant women in the city. Furthermore, the study will be used as a stepping stone for further studies to be conducted on other risk factors of LBW babies, pointing where to focus.

\section{Methods}

\section{Study area, design and period}


The research was conducted in randomly selected three government hospitals in Addis Ababa, Ethiopia, namely, Gandhi Memorial Hospital, Yekatit 12 Medical College Hospital, and Zewuditu Memorial Hospital. The determining factors of low birth weight among term babies born in government hospitals in Addis Ababa city were studied using a hospital-based unmatched case control study design. The study was carried out between June and September in the year 2020 G.C.

\section{Study population}

All randomly selected term babies (37 to 42 weeks) are delivered at three government hospitals in Addis Ababa. The cases were taken from neonates delivered in three government hospitals with a birth weight of less than or equal to $2,499 \mathrm{gm}$, while the controls were taken from those born with normal birth weight $(2,500 \mathrm{gm}$ to $4,200 \mathrm{gm})$. The eligibility criteria for participants were All birth cards (mother and neonate cards) with Low birth weight (less than or equal to $2,499 \mathrm{gm}$ ) or normal birth weight (2,500 gm to 4,200), Live birth, singleton term baby and Gestational age between 37 to 42 weeks. We exclude those mothers who had any chronic medical complications, eclampsia or pre-eclampsia, and babies with congenital abnormalities.

\section{Sample size determination}

The sample size (number of the cases) for the present study was determined using unmatched case control formula [15]. With the assumption of double population proportions formula. ANC visit, maternal weight during pregnancy, and gravida are considered. However, maternal weight during pregnancy that gives the maximum sample size of all; is finally taken as the main exposure variable to determine the sample size for the present study. Based on that, the percent of controls exposed ( $>50 \mathrm{~kg}$ ) is $4.7 \%$; Percent of cases with exposure is $12.5 \%$, both estimated from another study [16]. And, a 95\% Confidence Interval, $5 \%$ Level of Significance $(a)=0.05$ (two-sided), a Power of $80 \%$ and 2:1 allocation ratio of controls to cases are assumed. Using the formula, $151.9 \approx 152$ cases were calculated. Adding $10 \%$ non-response rates i.e. $\approx 16$; it became 168 . The controls were twice the cases so were 336 . And a total of 504participants were enrolled in the study.

\section{Sampling technique and procedure}

In Addis Ababa, there are 12 public hospitals. Obstetric and neonatology services are available at six of the institutions. Of these three hospitals i.e. Gandhi Memorial, Yekatit-12 Medical College, and Zewuditu Memorial Hospitals were selected by simple random sampling technique. Then, both the cases and controls were then chosen at random from term babies delivered at each of the three hospitals. The number of cases selected from each of the three hospitals is allotted proportionally based on the total sample size to the total number of women who gave birth (with relation to recent experience /in the previous three months prior to the data collection period). The number of controls chosen from each 
hospital was decided by the number of cases assigned to the given hospital. In that, the number of controls that were selected from a given hospital was twice that of the number of cases allotted to the hospital. Finally, the study units were selected using a systematic random sampling technique every $3^{\text {rd }}$ interval, after picking the first sampling unit at random using the list of medical record numbers and the registration book as a sampling frame.

\section{Data collection techniques and procedure}

The study was conducted in three governmental hospitals in the capital of Ethiopia, Addis Ababa. A total of 504 subjects ( 168 cases and 364 controls) were sampled using the large population formula. The data collection was carried out by record review and interviewing mothers using a document review checklist and a pre-tested structured questionnaire by phone call respectively. Trained BSc nurses collected the data, and the data collection process was supervised by health officers. For this purpose, two nurses at each hospital, or a total of six for all the three hospitals, and one Health Officer at each, or a total of three for all the three hospitals, were assigned to collect the data and supervise the data collection process, respectively. Overall, on every morning of the data collection period, the principal investigator went to the medical record unit of the hospital and examined the records of all birth cards for completeness of the records.

In this study, LBW was defined as a neonate with a birth weight of less than 2,500 grams. Through an interview using a structured questioners and checklist, the information was collected from the mother for socioeconomic characteristics and obstetrical history. A record review checklist was used for reviewing antenatal care cards. The weight and height of the mothers and birth weight of newborns were taken from the mothers'records. Caliberation was used to insure that the instruments used in the hospitals to weigh the mother and new born were up to the standard.

\section{Variables of the study}

\section{Dependent variables}

- Birth weight

\section{Independent variables}

- Socio-demographic characteristics, (newborn sex, maternal age, family monthly income, educational level, maternal occupation, religion, marital status, and others).

- Maternal and obstetrical characteristics (maternal weight, gravidity, parity, history of abortion, hemoglobin level, trimester of ANC visit, gestational age at birth, number of ANC follow up). 


\section{Data quality control}

Data quality was ensured during collection, coding, entry, and analysis. A structured checklist was used for reviewing the charts of mothers and neonates. To avoid confusion and to create shared understanding of the study, data collectors and supervisors received training on its purpose. Each card was checked for its completeness and appropriate documentation. Supervision of data collectors included observations on how the data collectors collected the data, checking the correctness and completeness of records, and vigilant examination of completed checklists and questionnaires for cleanness and quality of recording.

The data collectors were instructed to write the card number on the checklist during the data collection so that any identified errors were traced back using the card number. The filled checklist was checked for completeness by data collectors, supervisors, and investigators on a daily basis. Consequently, any problem encountered was discussed among the team and solved immediately. Above all, prior to the actual data collection process, all data collection tools and procedures were pretested for their capacity of to generate valid and valuable information as desired. The reliability of the tools was checked using Cronbach's alpha (0.897) during the pre-test.

\section{Data processing and analysis}

The data was entered and categorized using EPI Info.7 software. It was then imported into the statistical package for the social sciences (SPSS) version 24 software for further analysis. Descriptive statistics were used to describe demographic and socio economic characteristics of the study participants and the distribution of the risk factors for low birth weight among the cases and controls using frequencies and percentages. A bivariate logistic regression analysis was used to assess the possible independent effect of each independent factor on the dependent variable, with the respective crude odds ratio (COR) and $95 \%$ confidence interval. While multivariable logistic regression analyses were used for those independent variables with $<0.25 p$-values of the bivariate logistic regression analysis result, to assess their adjusted effects on the dependent variable, using the adjusted odds ratio (AOR) and $95 \%$ confidence interval. For all associations, throughout the analyses in the present study, P-values $<0.05$ were considered statistically significant.

\section{Operational definitions}

- Birth weight: is the first weight of the fetus or newborn obtained after birth, measured within the first hour of life before significant postnatal weight loss has been occurred by using a standard weight scale.

- Case: a neonate with a birth weight of less than 2, $500 \mathrm{gm}$.

- Control: a neonate with a birth weight 2, $500 \mathrm{gm}$. to 4, $200 \mathrm{gm}$. 
- Extremely low birth weight (severely underweight): is the weight of neonate less than 1,000 gm.

- Low birth weight (underweight): is the weight of neonate less than 2,500 gm.

- Normal birth weight: a neonate with a birth weight 2, $500 \mathrm{gm}$. to 4, $200 \mathrm{gm}$.

- Term baby: an infant born completing the full terms of pregnancy (37 to 42 weeks).

- Very low birth weight: is the weight of neonate less than 1,500 gm.

\section{Ethical consideration}

The ethical review committee of Debre Berhan University's College of Health Science gave their approval. It was also communicated to Addis Ababa City's Health Bureau. The Addis Ababa Public Health Research and Emergency Management Core Process, as well as the respective department heads of the obstetric ward in each of the three hospitals, gave their approval for the cards to be used. Since the cards contain the mothers' names, confidentiality was maintained by instructing the data collectors not to report any personal information contained on the cards. Following that, the mothers of the newborns were contacted via phone to obtain informed consent.

\section{Results}

\section{Socio demographic characteristics}

During the course of the study, 504 subjects were enrolled (168 cases and 368 controls) to participate in the study. The majority of our study subject mothers' age lies between 19 and 30 ( $54.2 \%$ of cases and $75.9 \%$ of controls). Most of the mothers were housewives, $72(42.8 \%)$ of cases and $132(39.3 \%)$ of controls. The majority of the mothers were married, $113(67.3 \%)$ of cases and $309(91.7 \%)$ of controls. About $59(35.1 \%)$ of cases and 120 (35.7\%) of control were government employed, while $30(17.9 \%)$ of cases and 65 (19.3\%) of control had private jobs. As it is shown in table 1, 75 (44.6\%) of cases and 192 $(57.1 \%)$ of controls have completed secondary school, while $18(10.7 \%)$ of cases and $23(6.8 \%)$ of controls were unable to read and write, and $32(19.0 \%)$ of cases and $37(11.0 \%)$ of controls have completed only primary school.

Significant numbers of our study subjects' mothers' weights were greater than $51 \mathrm{~kg}, 122(72.6 \%)$ of cases and $280(83.3 \%)$ of controls. (Table- 1$)$.

Table 1: Distribution of Socio-Demographic characteristics of Study participants in selected public Hospitals of Addis Ababa, Ethiopia, 2020 


\begin{tabular}{lll}
\hline Variable & Case $(\mathbf{N}, \%)$ & Control $(\mathbf{N}, \%)$ \\
\hline Maternal age & $36(21.4 \%)$ & $39(11.6 \%)$ \\
$\leq 18$ & $91(54.2 \%)$ & $255(75.9 \%)$ \\
$19-30$ & $41(24.4 \%)$ & $42(12.5 \%)$ \\
$>30$ & & \\
Occupation & $59(35.1 \%)$ & $120(35.7 \%)$ \\
Governmental employee & $30(17.9 \%)$ & $65(19.3 \%)$ \\
Private employee & $72(42.8 \%)$ & $132(39.3 \%)$ \\
House wife & $7(4.2 \%)$ & $19(5.7 \%)$ \\
Farmer & $113(67.3 \%)$ & $309(92.0 \%)$ \\
Marital status & $31(18.5 \%)$ & $16(4.8 \%)$ \\
Married & $24(14.3 \%)$ & $11(3.3 \%)$ \\
Not married & & \\
Divorced & & \\
& & \\
Educational status & $18(10.7 \%)$ & $23(6.8 \%)$ \\
Illiterate & $32(19.0 \%)$ & $37(11.0 \%)$ \\
Primary(1-6) & $75(44.6 \%)$ & $192(57.1 \%)$ \\
Secondary school(7-12) & $42(12.5 \%)$ \\
Diploma & $20(11.9 \%)$ & $42(12.5 \%)$ \\
Degree and above & $23(13.7 \%)$ & \\
Maternal Weight & & $56(16.7 \%)$ \\
$<50$ & $46(27.4 \%)$ & $280(83.3 \%)$ \\
>51 & $122(72.6 \%)$ & \\
& & \\
\hline
\end{tabular}

\section{Maternal and new born Characteristics}

In this study, the majority of cases $86(51.2 \%)$ were primi-parous, while significant numbers of controls were multiparous, $226(67.3 \%)$. In $232(69.0 \%)$ of controls and $83(49.4 \%)$ of cases, they had ANC followup more than 4 times, and in $270(80.4 \%)$ of controls and (90) $53.6 \%$ of cases, the first visit of ANC was in the first trimester of pregnancy. The majority of mothers in both groups 147 (87.5\%) of controls and 294 $(87.5 \%)$ of cases had nutritional advice from health professionals, and 303(90.2 \%) of controls, and $110(65.5 \%)$ of cases were supplemented with iron and folic acid. Half of the mothers in the case group $93(55.4 \%)$ and $258(76.8 \%)$ in the control group had no history of abortion prior to this pregnancy. Only 19 (5.7) and 26(15.5\%) of the mothers in the control and case groups, respectively, were alcoholics, and $4(2.4 \%)$ of cases and $2(0.6 \%)$ of controls were found to be smokers. In $284(84.6 \%)$ of controls and 122 (72.6\%) of cases, the hematocrit level ranges between 30 and $45 \%$. The pregnancy in $101(60.1 \%)$ of cases and $313(93.2 \%)$ of controls was wanted and planned, and $117(69.6 \%)$ of newborns in the case group and 165 (49.1\%) in the control group were females (Table 2). 
Table 2- Maternal and new born characteristics of Study participants in in selected public Hospitals of Addis Ababa, Ethiopia, 2020

\begin{tabular}{|c|c|c|}
\hline Variables & Cases (N, \%) & Controls (N, \%) \\
\hline \multicolumn{3}{|l|}{ Number of ANC visit } \\
\hline$\leq 3$ & $85(50.6 \%)$ & $104(31.0 \%)$ \\
\hline$\geq 4$ & $83(49.4 \%)$ & $232(69.0 \%)$ \\
\hline \multicolumn{3}{|l|}{$\bar{A} N C$ service } \\
\hline No & $32(19.0 \%)$ & $29(8.6 \%)$ \\
\hline Yes & $136(81.0 \%)$ & $307(91.4 \%)$ \\
\hline \multicolumn{3}{|l|}{ ANC service given during } \\
\hline 1 st trimester & $90(53.6 \%)$ & $270(80.4 \%)$ \\
\hline 2nd trimester & $38(22.6 \%)$ & $39(11.6 \%)$ \\
\hline 3rd trimester & $40(23.8 \%)$ & $27(8.0 \%)$ \\
\hline \multicolumn{3}{|l|}{ Sex } \\
\hline Male & $51(30.4 \%)$ & $171(50.9 \%)$ \\
\hline Female & $117(69.6 \%)$ & $165(49.1 \%)$ \\
\hline \multicolumn{3}{|l|}{ Abortion } \\
\hline No & $93(55.4 \%)$ & $258(76.8 \%)$ \\
\hline Yes & $75(44.6 \%)$ & $78(23.2 \%)$ \\
\hline \multicolumn{3}{|l|}{ Number of abortion } \\
\hline 0 & $93(55.4 \%)$ & $258(76.8 \%)$ \\
\hline$\leq 2$ & $72(42.9 \%)$ & $73(21.7 \%)$ \\
\hline$\geq 3$ & $3(1.8 \%)$ & $5(1.5 \%)$ \\
\hline \multicolumn{3}{|l|}{ khat chewing } \\
\hline No & 154(91.7\%) & $320(95.2 \%)$ \\
\hline Yes & $14(8.3 \%)$ & $16(4.8 \%)$ \\
\hline \multicolumn{3}{|l|}{ Alcohol consumption } \\
\hline No & $142(84.5 \%)$ & $317(94.3 \%)$ \\
\hline Yes & $26(15.5 \%)$ & $19(5.7 \%)$ \\
\hline \multicolumn{3}{|l|}{ Nutritional Advice } \\
\hline No & $30(17.9 \%)$ & $42(12.5 \%)$ \\
\hline Yes & $147(87.5$ & $294(87.5 \%)$ \\
\hline \multicolumn{3}{|l|}{ Iron and folic supplementation } \\
\hline No & $58(34.5 \%)$ & $33(9.8 \%)$ \\
\hline Yes & $110(65.5 \%)$ & $303(90.2 \%)$ \\
\hline \multicolumn{3}{|l|}{ Type of pregnancy } \\
\hline Wanted \& Planed & $101(60.1 \%)$ & $313(93.2 \%)$ \\
\hline Unwanted\& Unplanned & $67(39.9 \%)$ & $23(6.8 \%)$ \\
\hline \multicolumn{3}{|l|}{ History of Cigarette Smoking } \\
\hline No & 164(97.2\%) & $334(99.4 \%)$ \\
\hline Yes & $4(2.4 \%)$ & $2(0.6 \%)$ \\
\hline \multicolumn{3}{|l|}{ Number of Gravidity } \\
\hline Primi-Gravida & $77(45.8 \%)$ & $76(22.6 \%)$ \\
\hline Multi-Gravida & $91(54.2 \%)$ & $260(77.4 \%)$ \\
\hline \multicolumn{3}{|l|}{ Number of Parity } \\
\hline Primi & $86(51.2 \%)$ & $110(32.7 \%)$ \\
\hline Multi & $82(48.8 \%)$ & $226(67.3 \%)$ \\
\hline \multicolumn{3}{|l|}{ Hematocrit level } \\
\hline$<30$ & $46(27.4 \%)$ & $52(15.2 \%)$ \\
\hline $30-45$ & $122(72.6 \%)$ & $284(84.8 \%)$ \\
\hline \multicolumn{3}{|l|}{ Deworm drug } \\
\hline No & $155(92.3 \%)$ & $329(97.9 \%)$ \\
\hline Yes & $13(7.7 \%)$ & $7(2.1 \%)$ \\
\hline
\end{tabular}




\section{Association of different Characteristics of participant with Low Birth Weight}

In the bivariable analysis, all variables that were significantly associated with $p$ value $<0.25$ and $95 \% \mathrm{Cl}$ were entered into the multivariable analysis. Type of pregnancy, sex of baby, iron/folic acid supplementation, hematocrit level, mother's age $<18$, and lack of ANC visit were found to have a statistically significant association with low birth weight ( $p$-value $<0.05,95 \% \mathrm{Cl}$ ).

Mothers who had unwanted and unplanned pregnancies were more likely to deliver LBW neonates than mothers who had wanted and planned pregnancies (AOR: 1.25, 95\% Cl: 1.09, 2.66), mothers who had delivered female neonates were more likely to deliver LBW neonates than mothers who had delivered male neonates (AOR: 1.09, 95\% Cl: 2.04, 3.21), and the odds of low birth weight were higher among mothers who did not take iron/folic acidCompared to mothers aged 19-30, those under the age of 18 had a higher risk of having a LBW baby (AOR: 2.69, 95\% Cl: 1.24, 5.83). Neonates born to mothers with a hematocrit level of 30 were more likely to be LBW than those with a hematocrit level of 30-45 (AOR: 1.29, 95\% Cl: 1.01, 5.22). (Table 3).

Table 3: Bivariate and multivariate logistic regression analysis of factors associated with Low Birth Weight, in selected public Hospitals of Addis Ababa, Ethiopia, 2020 


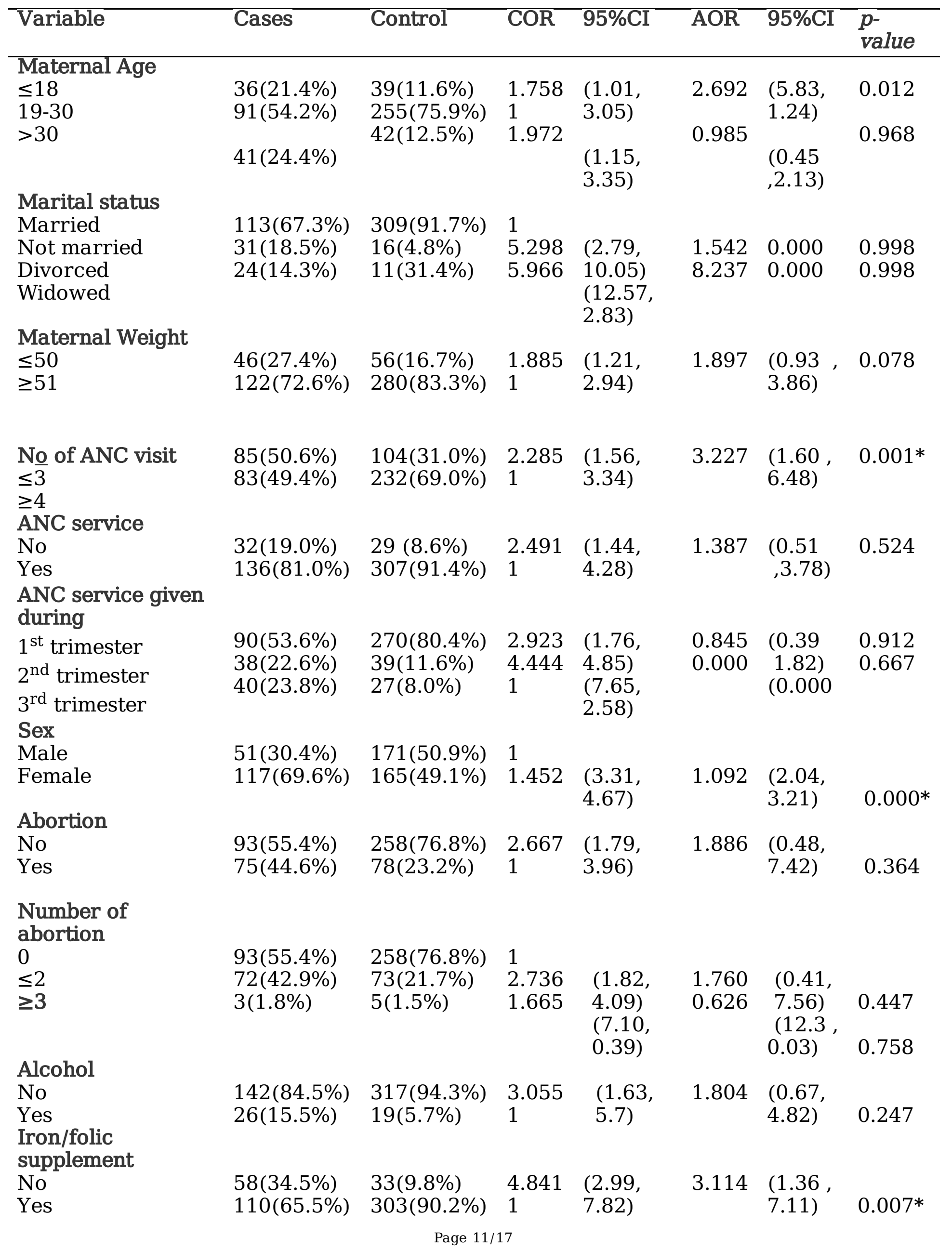


Type of pregnancy

Wanted\& Planed

$\begin{array}{llll}\text { Unwanted } & \text { 101(60.1\%) } & 313(93.2 \%) & 1\end{array}$

\&Unplanned

$67(39.9 \%) \quad 23(6.8 \%) \quad 2.475$

$(1.53, \quad 1.248 \quad(1.09$
$3.98)$

$0.005^{*}$

Gravidity

Primi-gravida

Multi-gravida

Parity

primi

multi

$77(45.8 \%)$

$76(22.6 \%) \quad 2.89$

91(54.2\%)

$260(77.4 \%) \quad 1$

$(1.94$

1.160

(0.38,

4.30)

3.46)

0.790

$86(51.2 \%)$

110(32.7\%) 2.155

(1.45,

$1.736(0.49$,

$82(48.8 \%$

226(67.3\%) 1

3.14)

6.06)

0.387

Hematocrit level

$<30$

30-45

Deworm drug

No

Yes

$46(27.4 \%)$

$122(72.6 \%)$

$52(15.2 \%)$

$284(84.8 \%) \quad 1$

2.059

3.22)

155(92.3\%)

$13(7.7 \%)$
$329(97.9 \%) \quad 1$

$7(2.1 \%)$
3.942
(1.31, $1.287 \quad(1.09$,

5.22)

0.049*

1.206 (0.34,

$(1.54$

10.07)

4.83)

0.772

Key: $\mathrm{COR}=$ Crude Odds Ratio, $\mathrm{CI}=$ Confidence Interval, $\mathrm{LB}=$ Lower Bound, $\mathrm{UB}=\mathrm{Upper}$ Bound, * Significant, AOR= Adjusted Odds Ratio

\section{Discussion}

In this study, it was found that maternal age below 18 was significantly associated with giving birth to low birth weight (AOR: $2.69,95 \% \mathrm{Cl}: 1.241,5.84)$. This was consistent with a study done by Gessese B. et al, in Mekelle hospital, Mekelle, Northern Ethiopia, which showed maternal age of 15 to 19 years had a statistically significant association with giving birth to LBW, with a p value of 0.001 . [21]). Another hospital-based case-control study conducted in the Bale zone of Eastern Ethiopia found that mothers under the age of 20 are more likely to have LBW newborns.[27]Studies done in the Sadar area of Bangladesh and the Ashanti Region of Ghana also showed that there is a statistically significant relation between maternal age and giving birth to LBW; ( $p$ values of 0.041 and 0.016 respectively). $[17,18]$. On the other hand, a study conducted in 2017 in Adwa, northern Ethiopia showed that mothers who were in the age group of less than 20 years were 1.7 more likely to deliver LBW babies than those in the age group of 21-35 years (AOR: 1.71, 95\% Cl: 2.16, 17.69). [19]. Contradict to this, a study conducted in 2015 in Iran showed, based on a chi-square test, that there is a significant correlation between the mothers' age (under 18 and above 35 years old) and low birth weight ( $p$ value of $\leq 0.001$ ) [20]. Similarly, a study done at Ribat University Hospital in Sudan revealed that maternal age, particularly extremes of age, has a significant effect on baby weight, with a significance value of 0.014 [21]. Another hospital based study conducted in Karnataka, India also showed that mothers aged 15-19 years had the highest prevalence of LBW (40\%), with a statistically significant association ( $p$ value of 0.010 ) [28]. An in-depth analysis of EDHS, 2011 by Tadesse Alemu and MelakuUmeta showed that as the maternal age group increased from 15-19 to 45-49, the risk of having LBW declined ( $p$ value below 0.05). (EDHS 2016). In a cross sectional study conducted 
in Zeinabeih hospital in Iran, it was revealed that maternal age below 18 years is significantly associated with LBW ( $p$ value of $\leq 0.001$ ), which is consistent with our results [22].

The other maternal factor found to be significantly associated with giving birth to LBW in this study was type of pregnancy (wanted and planned versus unwanted and unplanned) with a $p$ value of 0.005 . This result was consistent with findings from different literature. A cross-sectional study conducted in Zeinabeih hospital in Iran has revealed that an unwanted pregnancy could cause an increasing low birth weight ( $p$ value $<0.001$ ) [22]. A similar study conducted in Axum and Laelay Maichew districts, North Ethiopia, showed that mothers with unwanted and upland pregnancy were more likely to give birth to low birth weight newborns [AOR: 7.34, 95\% Cl: 2.05, 26.29]. [23] ).

Mothers who were anemic in their last pregnancy, mothers who did not take iron or folic acid supplementation are at increased risk of giving LBW babies with $p$ values of 0.049 and 0.007 , respectively. This was consistent with several pieces of literature. (Ghana in 2013, Khartoum in 2012, and Bangladesh in 2017) $[24,21,17]$.

Mothers who had fewer than three ANC visits were significantly associated with low birth weight (AOR: $3.227,95 \% \mathrm{Cl} ; 1.61,6.49)$. This is consistent with studies conducted by Rashidul A. et al. in their study to asses the distribution and determinants of LBW in developing countries, pointing out that inadequate antenatal care (ANC) (AOR: 1.7, 95\% Cl: 1.1, 2.8) had a significantly greater risk of delivering LBW babies [17]. And another survey conducted in 2018, in Gojjam, Debremarkos referral hospital, showed that the majority of cases $(73.7 \%$ ) had an ANC visit of 3 or less, whereas more than half of the controls $(59.6 \%)$ had four or more. And the final result indicated that there was a statistically significant association between the number of ANC visits and LBW (AOR: 3.81, 95\% Cl: 1.82, 7.99). [25]. A study conducted in Ghana in 2013 showed that the possibility of giving birth to children of low birth weight among women who do not receive ANC follow up is higher than those who receive antenatal care even once $29.0 \%$ versus $20.4 \%$ ). The result also showed that there was a highly significant association with LBW Antenatal Care ( $p$-value $\leq 0.001$ ) [26]. The 2016 EDHS survey showed that mothers who had never attended ANC follow-up had a $41 \%$ added risk of having low birth weight babies than those who had attended at least once during their last pregnancy (AOR: 1.41, 95\% Cl: 1.06, 1.88) [27].

This study has revealed that the sex of the newborn (females as compared to males) was significantly associated with low birth weight ( $p$ value of $\leq 0.001$ ). This is consistent with previous studies done in Bangladesh that indicated that female babies are more prone to being LBW than male babies [17]. In agreement with this, the present study indicated female babies had an increased odds of being Lbw, and another study in Mekelle hospital, in 2014, also showed that 128 (71.1\%) of new born babies with LBW were females. There was a statistically significant association between baby sex and BW; that is; female new born babies had a lower BW (1.74+-0.44) than male babies (1.95+-0.48). [28]

We used different strategies to enhance the strength of the study. First, to solve the recall bias, the information obtained by the phone call was cross-checked with the information available in the mother's card, and the reliability of the tools was checked using Cronbach's alpha (0.897) during the pre-test. The 
study has some limitations. For instance, the study only included mothers from three public hospitals, so it did not address those mothers from the other three public hospitals that also provide the service in the city administration. Furthermore, variables such as mothers' income and female size were not clearly labeled on the card, and the majority of the mothers were unwilling to answer questions during the phone interview. Again, variables that should be better taken in face-to-face contact by the involvement of professionals at all levels, the height of mothers was not easy to take via phone call, so potentially variables such as BMI were not included in this study.

\section{Conclusion}

The findings of this study showed that there is a significant association between some of the socio demographic status of the mothers and LBW newborns delivered in selected government hospitals in Addis Ababa. Among these socio demographic factors, maternal age below 18 years, unwanted and unplanned pregnancy, number of ANC visits below three in the last pregnancy, failure to be supplemented with iron and folic acid, and maternal hematocrit level have a statistically significant association with delivering an LBW newborn. The sex of the newborn also has a significant association with its birth weight. Female newborns are at higher risk of being LBW.

\section{List Of Abbreviations}

ANC: Antenatal Care; BW: Birth Weight; Cl: Confidence Interval; DB: Debre Berhan; EDHS: Ethiopia Demographic and Health Survey; ELBW: Extremely Low Birth Weight; GH: Gandhi Hospital; HIV: Human Immunodeficiency Virus; IUGR: Intra uterine Growth Retardation; LBW: Low Birth Weight; MUAC: Mid Upper Arm Circumference; NBW: Normal Birth Weight; OR: Odds Ratio; PIH: Pregnancy Induced Hypertension; SPHMMC: Saint Paulo's Hospital Medical Millennium College; TAH: Tikur Anbessa Hospital; TBH: Tirunesh Beijing Hospital; UNICEF: United Nations International Children Fund; VLBW: Very Low Birth Weight; WHO: World Health Organization; Y12H: Yekatit 12 Hospital; ZMH: Zewuditu Memorial Hospital; IMR: Infant Mortality Rate; IRB: Institutional Review Bored; VIF: Variable inflation factor.

\section{Declarations}

\section{Ethics approval and consent to participate}

Ethical approval and clearance was obtained from Debre Berhan University ethical review board. Informed consent was obtained from mothers, each study participants was informed about the aim of the study and participant involvement was on voluntary basis.

\section{Consent for publication}

Not applicable 


\section{Availability of data and materials}

The datasets used and/or analysed during the current study are available from the corresponding author on reasonable request.

\section{Competing interests}

The authors declare that they have no competing interests

\section{Funding}

This study has not received any fund

\section{Authors' contributions}

Y.A. involved from the inception to design, acquisition of data, analysis and interpretation, drafting the manuscript, M.K. involved in the acquisition, analysis, interpretation and revision of the manuscript, T.G. involved in the acquisition, analysis, interpretation and revision of the manuscript, M.H. involved in the acquisition, analysis, interpretation and revision of the manuscript, B.T. involved in the acquisition, analysis, interpretation and revision of the manuscript and E.T. involved in the acquisition, analysis, interpretation and revision of the manuscript. All authors read and approved the final manuscript.

\section{Acknowledgements}

We would like to acknowledge the School of Public Health, College of Health Sciences of Debre Berhan University for their material support. We will like to thank data collectors and study participants.

\section{Authors' information (optional)}

${ }^{1}$ Department of Preventive Medicine, College of Health Science, Addis Ababa University, Addis Ababa, Ethiopia. ${ }^{2}$ Department of Public Health, College of Health Science ,Debre Berhan University, Debre Berhan, Ethiopia. ${ }^{3}$ Armaeur Hansen Research Institute, Ministry of health, Addis Ababa, Ethiopia. ${ }^{4}$ Department of Human Nutrition, College of Medicine and Health Science, Kotebe Metropolitan University, Addis Ababa, Ethiopia.

\section{References}


1. UNCIEF, WHO, Low birth weight, country, regional and global estimates, Executive summary, New York, 2004.

2. UNICEF: Low Birth Weight New York, USA: United Nations Children's Fund; 2001.

3. WHO: Guide lines on optimal feeding of low birth weight infants in low and middle income countries. Geneva, Switzerland: World Health Organization 2011.

4. Hultman CM, Torrang A, Tuvblad C, Cnattingius S, Larsson JO and Lichtenstein P. Birth weight and attention-deficit/hyper activity symptoms in child hood and early adolescence: AProspective Swedish Twin Study. Journal of America Academic Child Adolescent Psychiatry 2007; 46: 370-7.

5. WHO: Low Birth Weight; Country, Regional and Global Estimates. Geneva, Switzerland \& New York, USA: World Health Organization \& United Nations Children's Fund 2004

6. Shrimp ton R.: Preventing low birth weight and reduction of child mortality. Trans R SocTrop Med Hyg 2003, 97(1):39-42.

7. UNICEF: Reduction of Low Birth Weight. Kathmandu, Nepal. United Nations Children's Fund Regional Office for South Asia 2002

8. World Bank: Repositioning Nutrition as Central to Development, A Strategy for Large Scale Action. Washington DC, USA 2006

9. Jammeh A, Sundby J, Vangen S.: Maternal and obstetric risk factors for low birth weight and preterm birth in rural Gambia: a hospital-based study of 1579 deliveries. Open Journal of Obstetrics and Gynecology 2011, 1, 94-103.

10. Feleke Y, Fikre E. Maternal age, parity and gestational age on the size of the new born in Addis Ababa. East African medical journal, 1999; 76(8).

11. Assefa B, Fikre E, Yusuf L. Birth to pregnancy interval and its effect on perinatal outcomes in Addis Ababa, Ethiopia. Ethiopian J. of Rep. Health, 2010; 4(1).

12. Kahsay Z, Tadesse A, Nigussie B: Low Birth Weight \& Associated Factors AmongNewborns in Gondar Town, North West Ethiopia: Institutional Based Cross Sectional Study, Indo Global Journal of Pharmaceutical Sciences, 2014; 4(2): 74-80.

13. Tema T. Prevalence and determinants of low birth weight in Jimma Zone, Southwest Ethiopia. East African Medical Journal 2006; 83:366-371.

14. Central Statistical Agency (CSA) [Ethiopia] and ICF (2011): Ethiopia Demographic and Health Survey 2011: Key Indicators Report. Addis Ababa, Ethiopia, and Rockville, Maryland, USA CSA and ICF

15. Kimk k: design and analysis of case-control studies. Seminar paper: November 16,2016.Acc.Feb.6/2018:Available at:www.ucdmc.ucdavis.edu/ctsc/

16. Demelash et al. risk factors of low birth weight in bale zone hospital, south east Ethiopia: A case control study, BMC Pregnancy and child birth (2015)15:264

17. Rashidual A, Marufa S, Abdur R distribution and determinants of low birth weight in developing countries in Dhaka Bangladesh, jprev med public health. 2017 jan;50(1):18-28 
18. R.Puurbalanta,A.O.Adebanji Determinants of low birth weight neonates: A case study of tamale Metropolis in Ghana. Journal for studies in management and planning , volume 01 issue 02 March 2015

19. Yisak.g, abera. H, Solomon.w, haftom.g The Prevalence and Risk Factors for Low Birth Weight among term Newborns in Adwa General Hospital, Northern Ethiopia. Obstetrics and Gynecology International Volume 2017, Article ID2149156,7 pages

20. Momeni M, Esfandyarpour R, Danaei M. The neglected socio behavioral risk factors of low birth weight SDH. 2015; 1(3):97-103..

21. Elmuntasir T, Mohamed A, Khalid N,Yousif M, Elsadig Y, Eiman El-Investigation of the Risk Factors of Low Birth Weight (LBW) in the National Ribat University Hospital, Khartoum .SUDANESE jOURNAL OF PUBLIC HEALTH -October 2012,vol.7 No.4

22. Victoria $C$, Mohammed $H$, SeyedM, Somayeh A , maternal risk factors associated with low birth weight,Iranian journal of health sci.2017;5(3):58-64

23. NegassiTeklehaimanot: Prevalence and Factors Associated with Low Birth Weight in Axum and LaelayMaichew Districts, North Ethiopia: A Comparative Cross Sectional Study

24. Radha.K, Ganga .B, Venkateswarvivm. A study on maternal factors affecting low birth weight in institutional deliveries department of community medicine ,Andhra medical college, Visakhapatnam .volly, Issuelverill (Jan 2015)page 45-48.

25. Sachin ,goirish.mrayesh.d, surekha.y, madhav.k,kiran.p maternal risk factors associated with term low birth weight neonates a matched pair case control study government medical college ,latur,manharastrsaindia 2011.

26. Michael O,*, Iddrisu A, Riskatu Y Maternal Risk Factors for Low Birth Weight in a District Hospital in Ashanti Region of Ghana Research in Obstetrics and Gynecology 2013, 2(4): 48-54

27. Taddese A and Melaku U, Meta Prevalence and predictors of small size babies in Ethiopia: in-depth Analysis of the Ethiopian demographic and health survey, 2011, Ethiop j health sci.2016 May; 26(3):243-250

28. Gessessew B, Balem D, Mussie A. Socio Demographic and Maternal Determinants of Low Birth Weight at Mekele Hospital, Northern Ethiopia: A Cross Sectional Study, American Journal of Advanced Drug Delivery. 2012. 